\title{
Calcul du mascaret sur machine électronique
}

\section{Tidal bore calculation on an electronic computer}

\author{
PAR
}

A. PREISSMANN

$\mathrm{ET}$

J.A. CUNGE

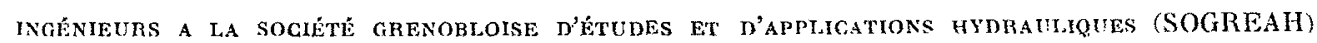

\begin{abstract}
Difficultés de principe rencontrées dans la résolution numérique des équations de SaintVenant par la méthode aux différences finies lorsque l'écoulement devient discontinu. Introduction d'une «pseudo-viscosité » reproduisant approximativement la perte de charge due au ressaut mobile et, donc, l'évolution du mascaret. Premiers calculs systématiques sur des exemples schématiques dégageant l'influence des divers facteurs qui peuvent influer sur la formation et la propagation du mascaret.
\end{abstract}

\begin{abstract}
Fundamental difficulties encountered in the numerical resolution of Saint-Venant's equations by the finite difference method, in the case of discontinuous flow conditions. Introduction of a "pseudo viscosity" as an approximate representation of the loss of head due to the travelling surge wave-and hence the development of the bore. Preliminary systematic calculations on simplified examples showing up the various factors liable to affect the formation and propagation of the bore.
\end{abstract}

\section{I. - INTRODUCTION}

La SOGREAH a établi divers programmes de calcul des intumescences dans les canaux et rivières qui, tous, sont basés sur la résolution en différences finies des équations de l'écoulement non stationnaire de Saint-Venant. Or, on sait que, dans certains cas, l'écoulement dans les canaux peut être discontinu. Le cas peut se présenter, par exemple dans les canaux d'amenée et de fuite d'usines hydroélectriques lors de manœuvres brusques. Dans ce cas, on observe des fronts d'onde extrêmement raides qui se propagent assez loin sans se déformer sensiblement. Remarquons que ce sont toujours les ondes positives qui sont raides. On constate que le front d'onde d'une onde négative s'étale toujours. On retrouve ces résultats par le calcul : en effet, on peut écrire les équations de la quantité de mouvement pour une discontinuité et on obtient une relation entre les divers paramètres, que nous appellerons la condition du ressaut, valable au voisinage de la discontinuité. On peut établir le bilan d'énergie et l'on constate, dans le cas d'une onde positive, que le ressaut doit détruire une certaine quantité d'énergie. Dans Je cas d'une onde négative, le calcul montre que si la discontinuité devait être maintenue, il faudrait que le ressaut fournisse une certaine quanlité d'énergie, ce qui est physiquement impossible.

Les ondes positives à front raide obéissent à des lois particulières comportant une perte de charge singulière à l'emplacement momentané de la discontinuité. II est donc évident a priori que les programmes ordinaires de calcul par différences finies ne sauraient convenir au cas de discontinuités.

Notons par ailleurs que les discontinuités peuvent intervenir spontanément dans un tronçon de canal, même lorsque les conditions aux limites qui définissent le problème ne présentent aucune discontinuité. C'est le cas précisément du mascaret.

L'apparition du mascaret est essentiellement dû à la non-linéarité des équations de SaintVenant. L'apparition d'une discontinuité est strictement impossible avec l'approximation linéaire.

Nous n'avons pas essayé, pour traiter les 
écoulements discontinus, une méthode qui consisterait à localiser par des tests la présence de discontinuités et de traiter par un sous-programme particulier les points de discontinuité.
Nous n'avons fait que reprendre une méthode utilisée récemment dans le problème analogue des ondes de choc des gaz compressibles, que nous avons adaptée à notre problème particulier.

\section{II. - L'ECOULEMENT DES GAZ EN CONDUITES ET L'INTRODUCTION D'UNE PSEUDO-VISCOSITÉ}

On connait depuis longtemps l'analogie entre l'écoulement de gaz en conduites et l'écoulement de l'eau dans les canaux.

Le phénomène du mascaret des écoulements en rivière correspond, dans la dynamique des gaz, aux ondes de choc caractérisées par une discontinuité de la pression et de la densité. Or, depuis quelque temps, on a utilisé pour l'étude des ondes de choc une méthode due à von Neumann et Richtmyer, que nous avons à peine modifiée de façon à l'adapter à l'étude de l'écoulement de liquides pesants dans un canal. Afin de simplifier l'écriture, nous allons écrire les équations du mouvement, d'une part pour un gaz dans une conduite de section constante, d'autre part dans un canal horizontal prismatique de section rectangulaire en négligeant le frottement. En coordonnées d'Euler, nous avons, dans le premier cas :

$$
\left.\begin{array}{l}
\left(\frac{\partial}{\partial t}+u \frac{\partial}{\partial x}\right) p=-\frac{\partial u}{\partial x} \\
\left(\frac{\partial}{\partial t}+u \frac{\partial}{\partial x}\right) u=-\frac{1}{\rho} \frac{\partial p}{\partial x} \\
\left(\frac{\partial}{\partial t}+u \frac{\partial}{\partial x}\right) \mathrm{E}=-\frac{p}{\rho} \frac{\partial u}{\partial x}
\end{array}\right\}
$$

- représente la densité du gaz;

$u$ sa vitesse d'écoulement;

$p$ sa pression;

$\mathrm{E}$ son énergie interne spécifique.

Dans le deuxième cas, nous avons :

$$
\left.\begin{array}{l}
\left(\frac{\partial}{\partial t}+v \frac{\partial}{\partial x}\right) h=-h \frac{\partial v}{\partial x} \\
\left(\frac{\partial}{\partial t}+v \frac{\partial}{\partial x}\right) v=-\frac{1}{h} \frac{\partial g\left(h^{2} / 2\right)}{d x}
\end{array}\right\}
$$

$h$ représente la profondeur de l'eau; $v$ la vitesse d'écoulement.

On constate l'analogie entre les systimes (1) et (2) en faisant correspondre $u$ à $v, \rho \dot{a} h$ et $p$ à $g\left(h^{2} / 2\right)$. Quant à la troisième des équations (1), elle exprime que l'augmentation de l'énergie interne d'une masse de fluide est égale au travail extérieur. En faisant correspondre $\mathrm{E}$ à $(g h / 2)$ (énergic potentielle), l'analogue de la troisième équation (1) est identiquement satisfaite. Dans le système (1), il est possible de se passer de la troisième équation en admettant que $p$ est une fonction univoque de e. Dans notre analogie cela correspondrait à la loi $p v^{2}$.

Les lois (1) et (2) sont valables lorsque le mouvement est continu; lorsqu'une discontinuité intervient, il est possible d'écrire les équations relatives à la continuité de la masse et à la continuité de la quantité de mouvement. Par exemple, pour les discontinuités en canal, on trouve la condition (l'indice 1 se rapporte à l'aval, l'indice 2 à l'amont) :

$$
v_{2}-v_{1}=\sqrt{\left(\frac{g h_{2}^{2}}{2}-\frac{g h_{1}^{2}}{2}\right)\left(\frac{1}{h_{1}}-\frac{1}{h_{2}}\right)}
$$

pour une discontinuité se déplacant à la vitesse

$$
c=\frac{h_{2} v_{2}-h_{1} v_{1}}{v_{2}-v_{1}}
$$

Il convient d'observer que, dans le cas de la discontinuité, il n'est pas possible d'assimiler $E$ à l'énergie potentielle $(g h / 2)$ sans enfreindre la loi de conservation de l'énergie. Le mouvement discontinu est accompagné d'une dissipation qui n'existe pas en mouvement continu. On conçoit donc que l'utilisation d'un programme de calcul prévu pour le mouvement continu donne des résultats aberrants lorsqu'une discontinuité se présente.

On pourrait naturellement tenter en cours de calcul de repérer la discontinuité et d'appliquer comme condition de passage la formule (3), la formule (4) permetlant de délerminer le déplacement de la discontinuité pour un intervalle de temps donné et de préparer ainsi le pas de calcul suivant. Nous n'avons pas suivi celle voie qui conduit certainement à des complications sensibles dans la programmation.

L'idée de von Neumann et Richtinyer consiste à introduire dans les équations (1) ou (2) un terme supplémentaire (pseudo-viscosité) qui :

-... n'affecte que très peu l'écoulement dans les parties où il reste continu; 


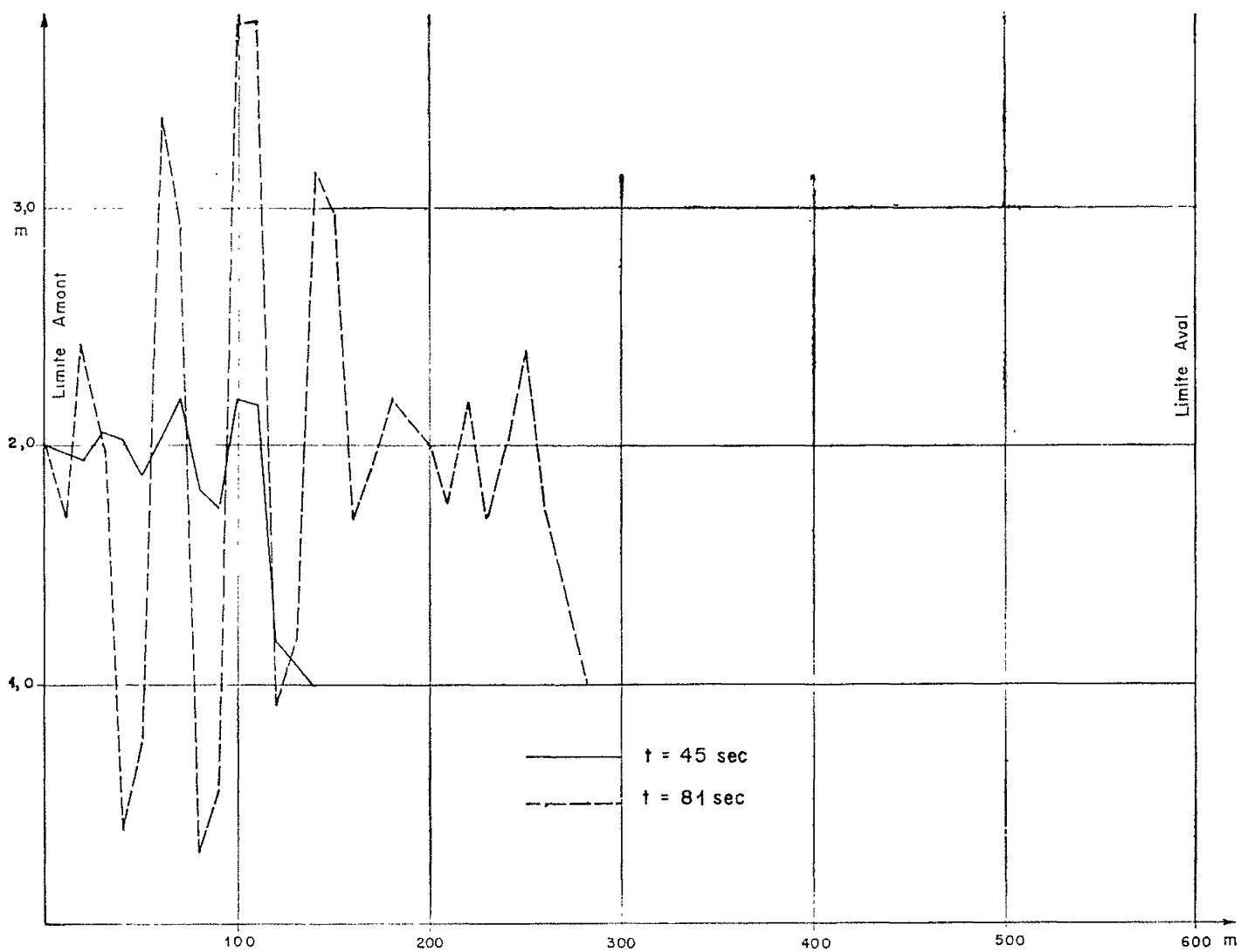

FIG. 1

Calcul d'une onde à front raide sans pseudo-viscosité.

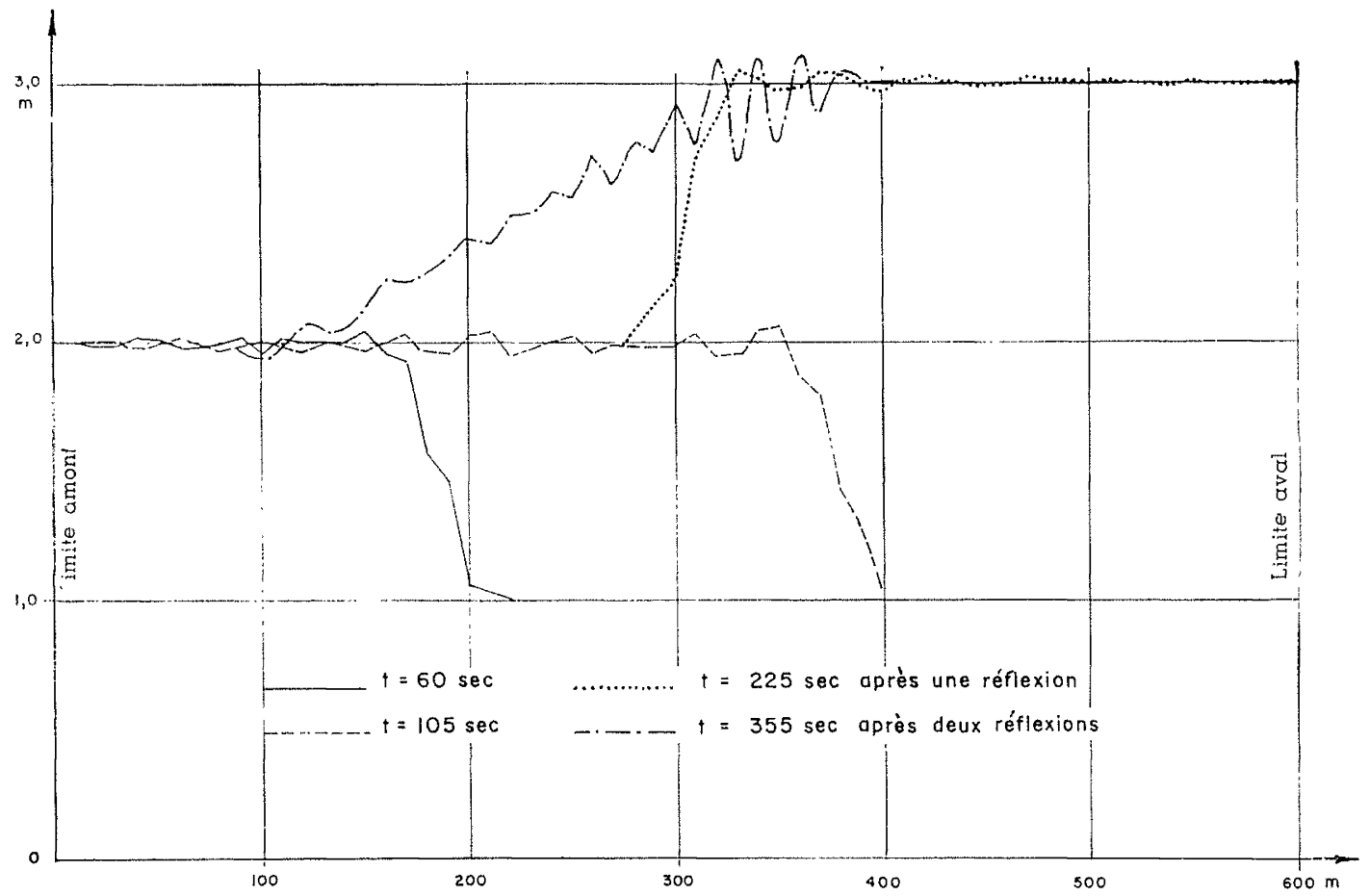

FIG. 2

Calcul d'une onde à front raide avec pseudo-viscosité. 
- provoque la perte de charge correcte aux discontinuités.

Les équations (2) prennent alors la forme :

$$
\begin{aligned}
& \left(\frac{\partial}{\partial t}+v \frac{\partial}{\partial x}\right) h=-h \frac{\partial v}{\partial x} \\
& \left.\left(\frac{\partial}{\partial t}+v \frac{\partial}{\partial x}\right) v=-\frac{1}{h} \frac{\partial\left[\left(g h^{2} / 2\right)+\eta\right]}{\partial x}\right)
\end{aligned}
$$

Le terme additionnel de pseudo-viscosité est donné par :

$$
q=\left\{\begin{array}{cl}
l^{2} h\left(\frac{\partial v}{\partial x}\right)^{2} & \text { si } \frac{\partial v}{\partial x}<0 \\
0 & \text { si } \frac{\partial v}{\partial x} \geqslant 0
\end{array}\right.
$$

Les calculs théoriques effectués — pour l'écoulement des gaz - confirment la possibilité d'utiliser la pseudo-viscosité pour reproduire approximativement les écoulements discontinus, En fait, les écoulements qui résultent du calcul sont des écoulements continus. Au lieu d'une discontinuité franche, la variation de pression se manifeste sur une longueur de l'ordre de grandeur du coefficient $l$ introduit de facon arbitraire dans l'expression de la pseudo-viscosité.

Si on veut résoudre les équations $\left(2^{\prime}\right)$ par différences finies, il semble justifié de choisir

$$
l=n \Delta x \text {. }
$$

- $n$ étant égal à 2 ou 3 ;

- $\Delta x$ représentant l'intervalle de longueur.
Afin d'expérimenter la méthode dans le cas de la propagation d'intumescences, nous avons procédé au calcul d'un cas schématique en modifiant légèrement notre programme général par l'introduction du terme de pseudo-viscosité. Il s'agit d'un canal rectangulaire prismatique horizontal long de $600 \mathrm{~m}$ et de $1 \mathrm{~m}$ de large. La résistance à l'écoulement était posée égale à zéro.

Les conditions initiales sont caractérisées par une profondeur constante de $1 \mathrm{~m}$ et une vitesse également constante de $1 \mathrm{~m} / \mathrm{s}$. Nous avons imposé à l'amont du canal le maintien du débit à $1 \mathrm{~m}^{3} / \mathrm{s}$ et à l'aval une montée relativement rapide de la profondeur de $1 \mathrm{~m}$ à $2 \mathrm{~m}$, puis son maintien à cette valeur. Les résultats sont consignés dans les figures 1 et 2. La figure 1 montre les résultats qu'on obtient avec le programme habituel. On remarque que lorsque le front d'onde devient raide, il apparaît des oscillations importantes du niveau amont; le calcul n'a pas $\mathrm{pu}$ être poursuivi du fait que la profondeur serait devenue négative. La figure 2 montre les résultats obtenus avec pseudo-viscosité : si la discontinuité n'est pas franche, le caractère essentiel du phénomène est conservé. Les petites oscillations parasites de part et d'autre de la discontinuité sont inhérentes au procédé utilise; elles interviennent également dans les calculs effectués par von Neumann et Richtmyer. Les réflexions aux extrémités du canal donnent les résultats attendus. Notons, après réflexion à l'amont du canal, une onde négative qui ne peut pas se maintenir raide. L'intervalle de temps choisi pour le calcul par différences finies est de $1,5 \mathrm{~s}$, l'intervalle de longueur de $10 \mathrm{~m}$.

\section{III. - ESSAIS D'ÉTUDE DU MASCARET SUR CALCULATRICE ELECTRONIQUE}

En principe, l'introduction de la pseudo-viscosité doit permettre, sans grandes difficultés de programmation, de traiter le cas d'écoulements discontinus dans des cas concrets lorsque le frottement rugueux est important et que les sections varient de forme. Toutefois, il nous faut constater que l'application à l'étude du mascaret du calcul sur machine électronique demande un travail de détail considérable. Nous ne pouvons donc donner ici que des premiers résultats très fragmentaires qui permettent cependant de tirer quelques conclusions d’ordre général.

Les premiers calculs effectués ont eu pour but de montrer l'importance de la rugosité du fond sur l'apparition et la propagation du mascaret. Nous avons pour cela traité le cas d'un canal rectangulaire de largeur variable, représenté en plan et en coupe longitudinale sur la figure 3 .
La longueur totale du canal est de $450 \mathrm{~km}$; seuls en réalité nous intéressent les $230 \mathrm{~km}$ aval, l'adjonction du canal à forte pente de $230 \mathrm{~km}$ ayant pour seul objet d'éviter une réflexion du mascaret. Le coefficient de rugosité de Strickler de la partie amont du canal a été fixé à

$$
40 \frac{\mathrm{m}^{3 / 3}}{\mathrm{~s}}
$$

celui de la partie aval a été choisi différent dans trois essais de calcul. A l'amont, nous avons imposé un débit constant. La marée donnant la condition aval a été choisie sinusoïdale, la profondeur variant entre $2 \mathrm{~m}$ et $8 \mathrm{~m}$ avec une période 12 heures. L'étal initial choisi correspondait à un écoulement permanent. Afin d'obtenir des résultats significatifs, nous avons naturellement dû prolonger le calcul et calculer sur 


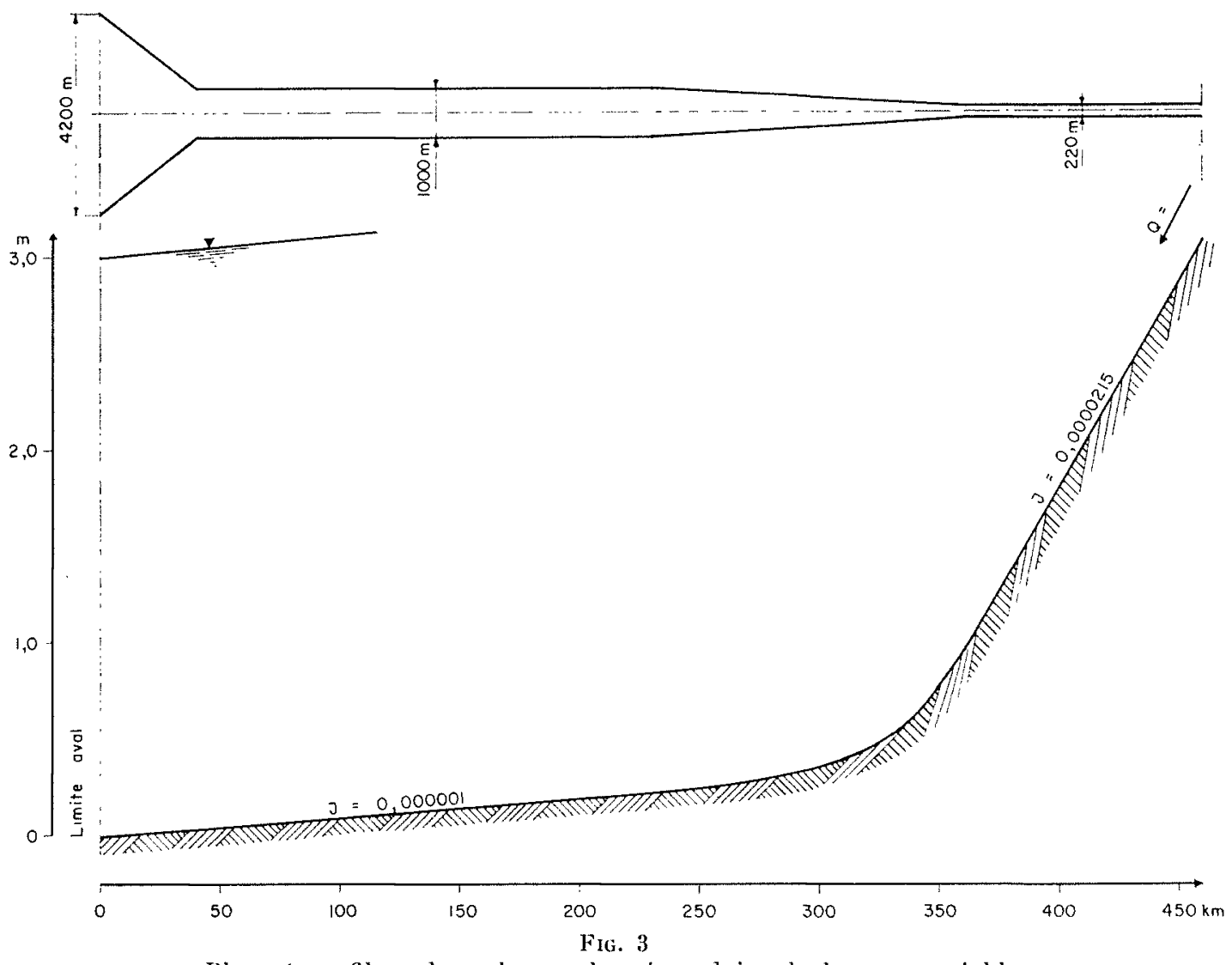

Plan et profil en long du canal rectangulaire de largeur variable.

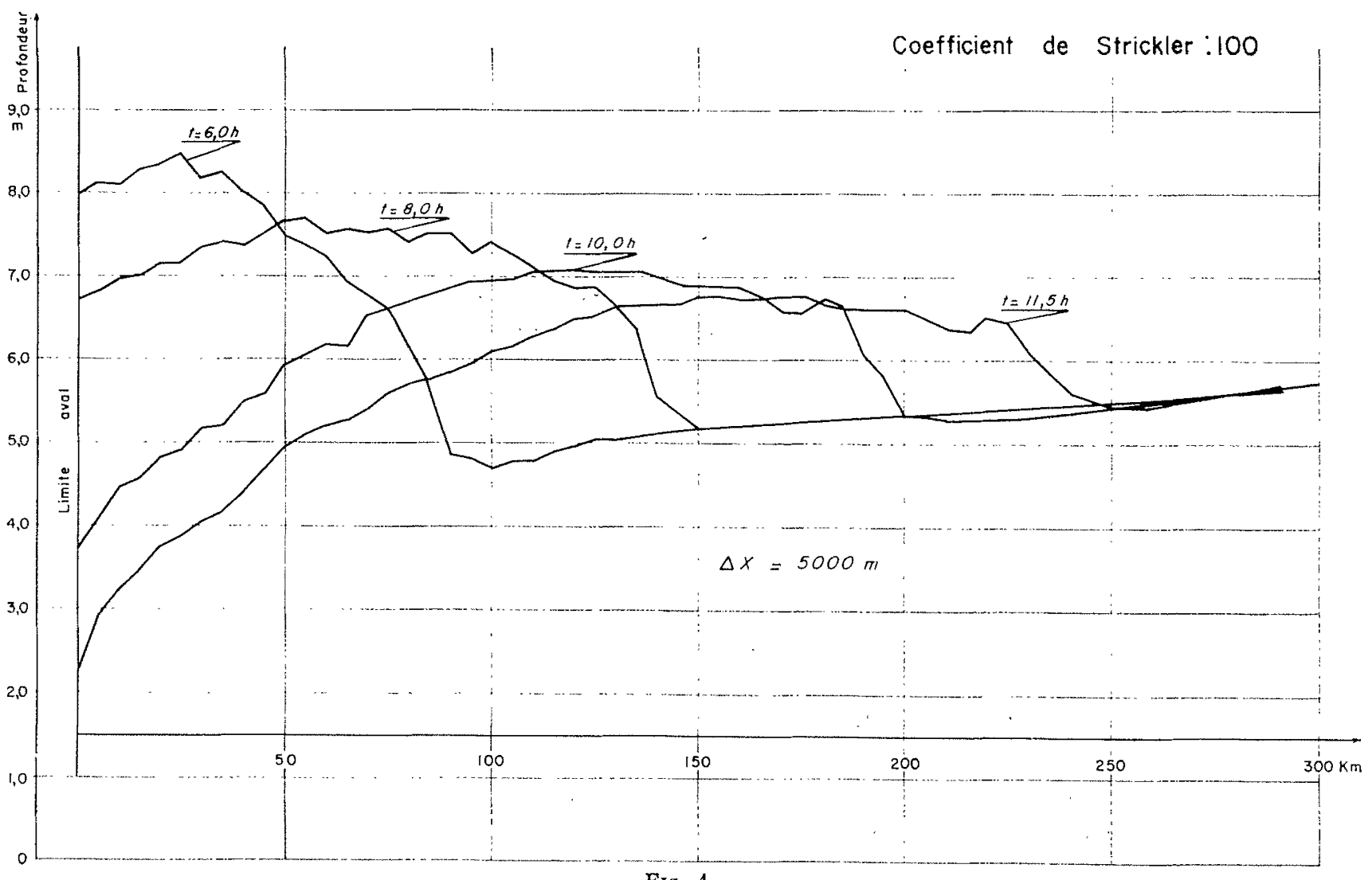

Fig. 4

Influence de la rugosité sur la propagation du mascaret

dans un canal rectangulaire de largeur variable. 


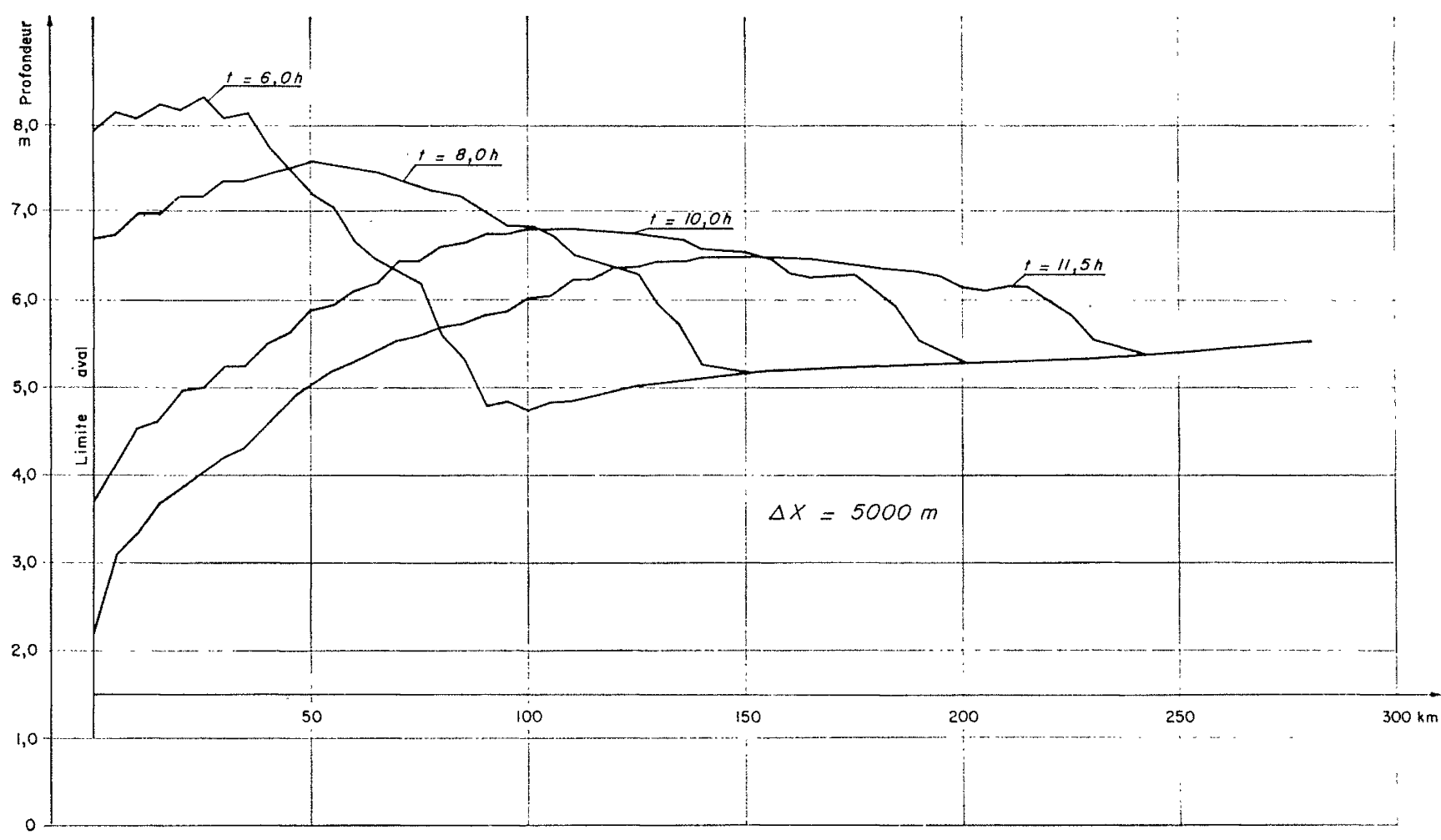

Fig. 5

Influence de la rugosité sur la propagation du mascaret dans un canal rectangulaire de largeur variable.

Coefficient de Strickler : 60

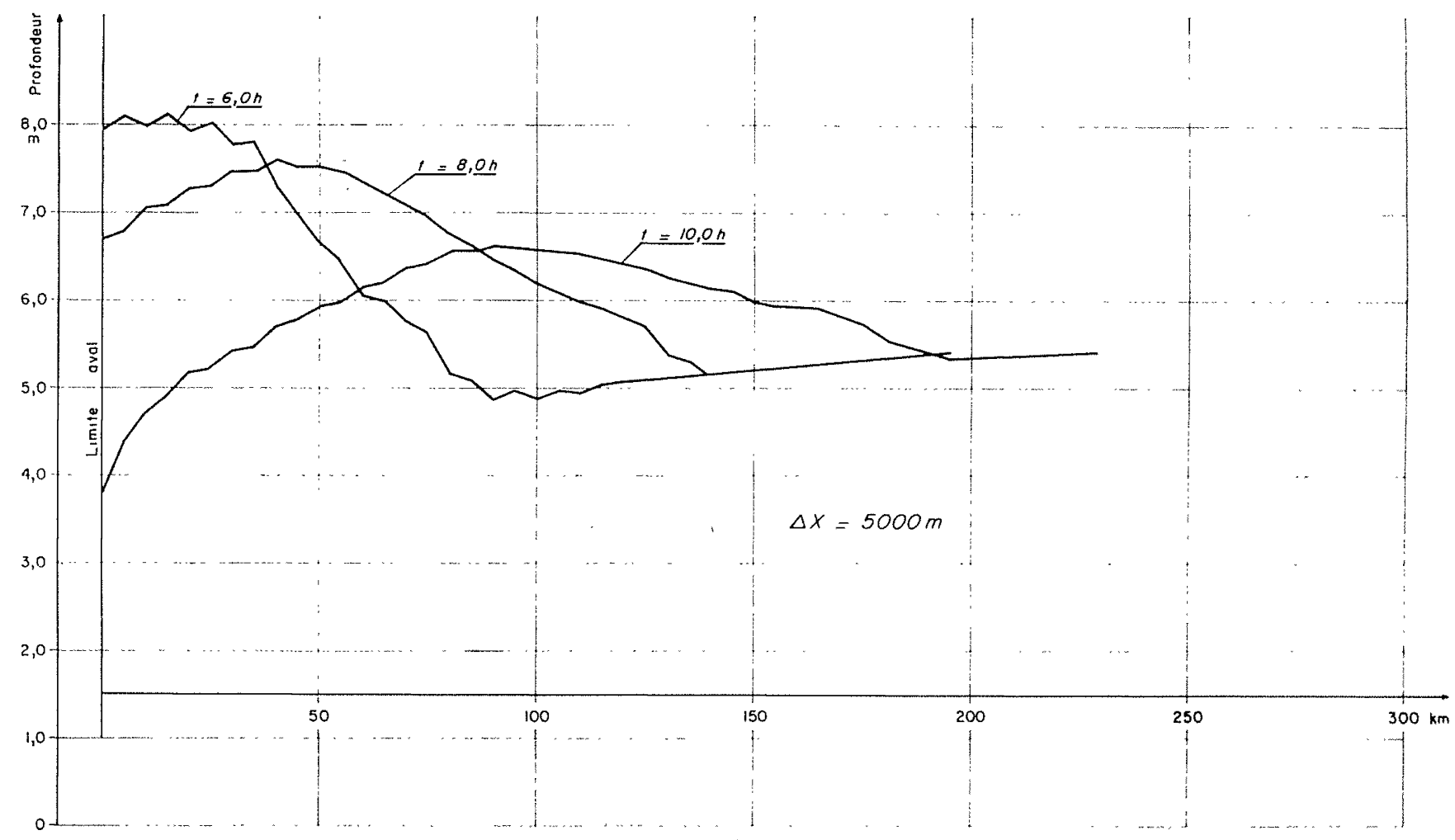

Fig. 6

Influence de la rugosité sur la propagation du mascaret dans un canal rectangulaire de largeur variable. 


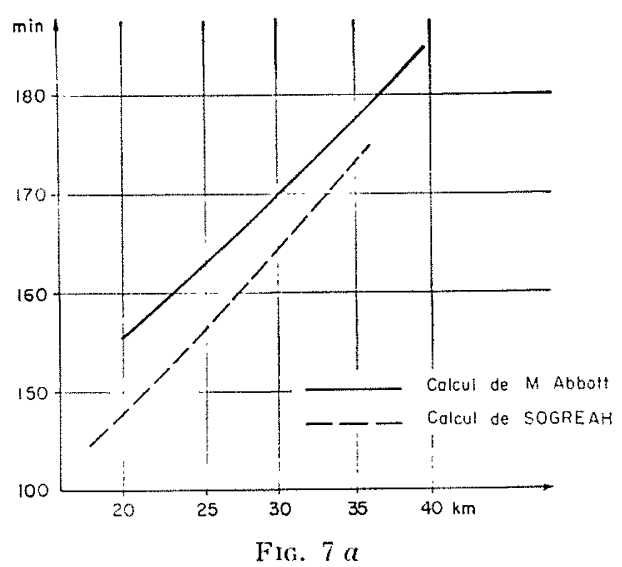

Cheminement du mascaret dans la Severn River.

une durée de 36 heures, c'est-à-dire sur trois marées complètes. Nous avons constaté que cette durée était suffisante, en effet les résultats pour la deuxième et la troisième marées concordent pratiquement.

Pour éviter une trop longue durée du calcul, nous avons choisi un intervalle de longueur $\Delta x$ de $5 \mathrm{~km}$.

Il a été effectué trois essais pour trois valeurs différentes du coefficient de rugosité $k$ de Strickler du tronçon aval. Les figures 4,5 et 6 montrent des lignes d'eau à différents moments pour les valeurs $k=100, k=80$, et $k=60 \mathrm{~m}^{1 / 3} / \mathrm{s}$. On remarquera que le mascaret se forme et se propage pour les faibles rugosités, alors que pour $k=60 \mathrm{~m}^{1 / 3} / \mathrm{s}$, il n'est pas possible de déceler les discontinuités.

Il semble donc que, lors de la reproduction du mascaret, soit par un modèle mathématique, soit par un modèle réduit hydraulique, il soit fort important de reproduire la perte de charge naturelle. Naturellement, les recherches relatives à l'influence de la rugosité sur l'apparition et la propagation du mascaret devront être poursuivies. En particulier le choix de l'intervalle de longueur $\Delta x$ introduit dans le calcul aux diffé- rences a été dicté par des considérations d'économie; la discontinuité apparaîtrait certainement mieux si l'on avait pu choisir une valeur sensiblement plus faible.

Afin de traiter un cas qui ressemble à un cas pratique, nous avons repris un travail de M. Abbott relatif à la propagation du mascaret dans l'estuaire de la Severn. En schématisant assez fortement la forme des sections de la Severn, nous avons procédé au calcul en prenant un intervalle de longueur égal à $1 \mathrm{~km}$. Les résultats que nous obtenons semblent assez bien confirmer les vues théoriques de M. Abbott sur la propagation du mascaret. En particulier, la figure 7 a montre le cheminement de la discontinuité. En abscisse, on reporte la distance comptée de l'aval, en ordonnée, l'intervalle de temps entre la basse mer et l'apparition du mascarel. La figure $7 b$ montre l'évolution de la hauteur du mascaret en fonction de l'emplacement. On constate la concordance entre notre calcul et celui de M. Abbott.

Evidemment, il ne s'agit encore que d'une schématisation. Notre calcul a été effectué avec une valeur $k=60 \mathrm{~m}^{1 / 3} / \mathrm{s}$. Pour pousser le calcul plus loin, il nous faudrait introduire des données plus complètes en ce qui concerne la forme des sections, la rugosité, la courbe de marée (existences d'harmoniques).

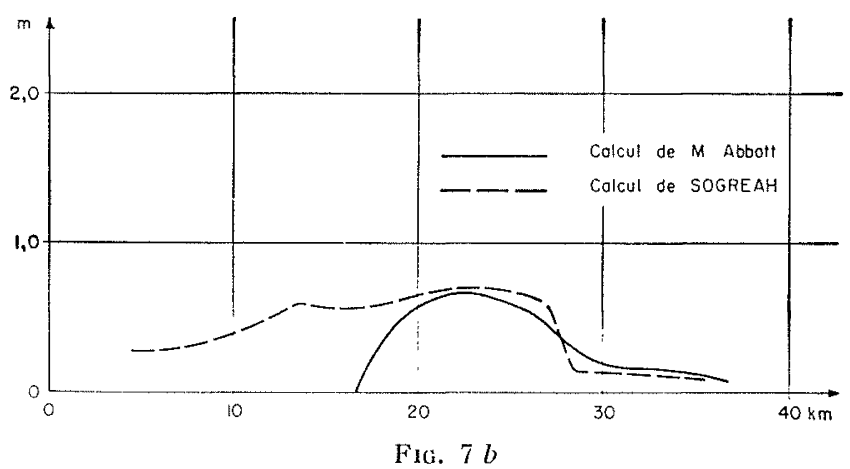

Hauteur du mascaret dans la Severn liver.

\section{IV. - CONCLUSIONS}

Il semble bien que l'introduction de la pseudoviscosité soit un moyen pratique de traiter les écoulements discontinus. Toutefois, l'expérience que nous avons dans ce domaine n'est pas encore suffisante pour tirer des conclusions définitives.

Nous pensons continuer nos essais de calcul dans deux directions:

1. Nous continuerons les essais systématiques destinés à déterminer l'influence de divers facteurs sur l'apparition et la propagation du mas- caret : rugosité, forme de l'estuaire, présence d'harmoniques dans la courbe de marée;

2. Nous tenterons de reproduire par le calcul le mascaret naturel dans quelques cas typiques, de façon à nous assurer qu'il est possible, en utilisant les données topographiques et hydrographiques, de reproduire l'évolution réelle du mascaret.

A ce sujet nous devons constater que l'Ordinateur IBM 650 dont dispose actuellement la 
SOGREAH et qui est d'une rapidité moyenne parmi les calculateurs électroniques, n'est pas suffisant pour permettre une investigation vraiment systématique. En effet, dans la méthode von Neumann et Richtmyer, il convient de choisir l'intervalle de longueur $\Delta x$ assez petit, de facon à bien localiser l'emplacement du mascaret ce qui oblige également à choisir un intervalle de temps $\Delta t$ également faible. Le temps de calcul est loin d'être négligeable. L'utilisation de calculateurs électronicues plus rapides semble donc s'imposer.

\section{Bibliographie}

[1] H. Favre. - Etude théorique et expérimentale des ondes de translation dans les canaux découverts. Dunod, Paris, 1935.

[2] R. D. Richtmyer. - Difference methods for initial- value problems. Interscience Publishers, New York, 1957.

[3] M.R. Asiotr. - A theory of the propagation of bores in channels and rivers. Proceedings of the Cambridge Philosophical Society, vol. 52, 1956 .

\section{I S C U S S I O N}

Président : M. Chapouthier

M. le Président remercie M. Preissmann pour son exposé très intéressant et nouveau pour certaines persomnes et dit qu'il a étẻ surpris de constater que le calcul donne un étalement aussi rapide du mascarel car la diminution de la profondeur et le rétrécissement du canal Iui auraient fait penser, qu'au contraire, il se raidissait. Cela a-t-il été vérifié expérimentalement?

M. Preissmann ne peut le dire car il n'a pas fait luimême d'expérience à ce sujet.

M. Banal dit que l'effet de la propagation sur le mascaret diffère suivant les circonstances. Cet effet est le résultat de deux actions contradictoires : une qui renforce le mascaret, l'autre qui l'affaiblit et qui fait que le marnage même disparait.

M. Preissmann pense que les pertes de charge ont pour effet d'aplanir le mascaret qui peut disparaître d'un coup.

M. le Président remarque que ceci est contradictoire avec les exposés précédents comme celui de M. NouGARo où il apparaît que les frottements et la rugosité du lit ont peu d'importance dans l'évolution du phénomène.

M. Remenieras souligne qu'ici il $y$ a une influence de la perte de charge sur une sorte de discontinuité.

M. Preissmann dit que les conclusions auxquelles il est arrivé sont un peu provisoires.

M. DE Rouville mentionne que, lorsque l'on a amélioré une rivière et que la marée s'y propage mieux, le mascaret disparaît (cas de la Seine).

M. Chapon pense qu'en fait le mascaret ne disparaît pas complètement dans toute la rivière mais se déplace; le mascaret de la Seine apparaissait autrefois dans la partie aval de la rivière mais depuis que l'on a approfondi la partie comprise entre Quillebeuf et Caudebec, il a disparu dans cette zone et s'est déplacé vers l'amont jusqu'au voisinage de Rouen. En ce qui concerne l'intervention du frottement en Seine, M. CHApon pense qu'elle est très importante et est en relation avec la célérité; en effet, dans le cas du mascaret la célérité est comprise entre 25 et $30 \mathrm{~km} / \mathrm{h}$ et n'atteint jamais les valeurs de $100 \mathrm{~km} / \mathrm{h}$ dont il a été parlé pour les intumescences; on peut se demander si l'influence mème du frottement ne varie pas avec la célérițé de l'onde.

M. Preissmane, pour répondre à celte remarque, dit que lorsque l'on approfondit une rivière, il $y$ a deux influences :

- Initialement l'intumescence, à l'endroit de la marée, impose une vitesse $\sqrt[2]{g} \mathrm{H}$ plus grande qu'à tel autre endroit; l'approfondissement crée un rattrapage qui sera d'autant plus rapide que la prolondeur initiale est faible;

- D'autre part l'approfondissement diminue l'étalement dû aux pertes de charge.

M. Chapon précise que, dans le phénomène de propagation du mascaret dans une rivière, il faut faire intervenir les harmoniques dont l'apparition est en partie liée au frottement. Il faut considérer en particulier la phase des harmoniques par rapport à l'onde fondamentale: si tous les harmoniques sont calés en phase sur le fondamental, il y aura un raidissement du front de l'onde et l'apparition des ondulations qui suivent le front de l'onde. Dans le cas de la Seine, une thèse soutenue récemment par M. le Flocl du Creo sur la propagation des marées en Seine a abouti a une décomposition harmonique du coefficient de frollement qui aurait ainsi des valeurs liées aux harmoniques de l'onde clle-même; il semble donc que pour les ondes de faible célérité, le frottement ait une inlluence non négligeable - contrairement aux intumescences de grande célérité.

M. le Président dit qu'il ne s'agit pas du méme phénomène et, passant à un autre aspect demande ce que devient la pseudo-viscosité dans le cas de l'onde négative.

M. Preissmann répond qu'elle disparait presque automatiquement.

Il semble à M. Charrueav que la pseudo-viscosité correspond au remplacement d'une onde de choc dans laquelle la discontinuité est une surface, par une quasi 
onde de choc dans laquelle la discontinuité se propage suivant un certain volume compris entre deux surfaces. Le choix de cette distance entre les deux surfaces est-il arbitraire?

M. Preissmann dit que, comme l'ont montré von Neumann et Richtmyer, cette distance est choisie pour la convenance des calculs. Il ne faut ni trop ni trop peu étaler cette discontinuité.

II. Thmniot demande comment on ajuste la psseudoviscosité.

M. Preissiann r'épond qu'on ne l'ajuste pas. Dans l'équation :

$$
q=(n \Delta x)^{2} h\left(\frac{d y}{d x}\right)^{2}
$$

on choisit $n$, le seul terme inconnu, égal à 2 ou 3. II n'y a donc pas d'ajustement, $\Delta x$ est uniquement une distance de calcul.

M. Crvae ajoute que, lorsqu'on prend $n$ supérieur à 3 , il $y$ a des oscillations parasites. Un autre coefficient qui varic proportionnellement avec les carrés de la discontinuité, intervient.

M. Preissmann indique que l'on n'a pas de discontinuité, lorsqu'il n'y a pas de risque que les ondes se rattrapent, $d x$ est pratiquement nul par rapport aux autres termes.

M. le Président, pour répondre à la question de M. Thirniot et en résumant ce que viennent de dire M. Preissmann et M. Cunge, dit qu'il y a tout de même un certain ajustement physique implicite.

M. Faune dit que l'influence de la rugosité est forte (cf. fig. 9 de la communication de M. NAFAS). D'autre part, dans une étude sur l'estuairc de la Gironde, il a calculé, par la méthode des caractéristiques, l'instant et le lieu de formation du mascaret. Sur le modèle réalisé et dans la nature on constatait que le mascaret se produisait, par exemple, dans le bras de Macau plus fréquemment lorsque les fonds étaient hauts.

En réponsc à M. Thirniot, M. Preissmane indique que le coefficient $n$ reste fixé à 2 ou 3 et que son étude reste au stade des mathématiques expérimentales ce qui a un inconvénient car rien n'est vérifié par l'expérience.

M. Preissmann compte reprendre des expériences déjà faites et essayer de leur appliquer sa méthode de calcul.

M. REMENieras signale qu'il a étudié au Laboratoire de Beauvert de la S.H.F. la propagation d'une onde de translation dans un canal convergent à berges verticales (1).

Bien que le phénomène soit assez différent de celui du mascaret on constate une forte augmentation de la hauteur du corps de l'onde lorsqu'elle se déplace vers l'extrêmité rétrécie du convergent. La valeur de cette augmentation a pu ètre calculée analytiquement moyennant certaines hypothèses simplificatrices; ce calcul pourrait sans doute être repris avec plus de précision (prise en compte des pertes de charge) par la méthode exposée par MM. Preissmann et Cunge.

M. le Président remercie M. Preissmann et M. Cunge pour Icur communication.

(1) G. Remenifras. - Augmentation de la hauteur d'une onde de translation se déplaçant dans un canal convergent - La Houille Blanche $n^{\circ}$ 6 - 1957 (IVo Journées de 1'Hydraulique).

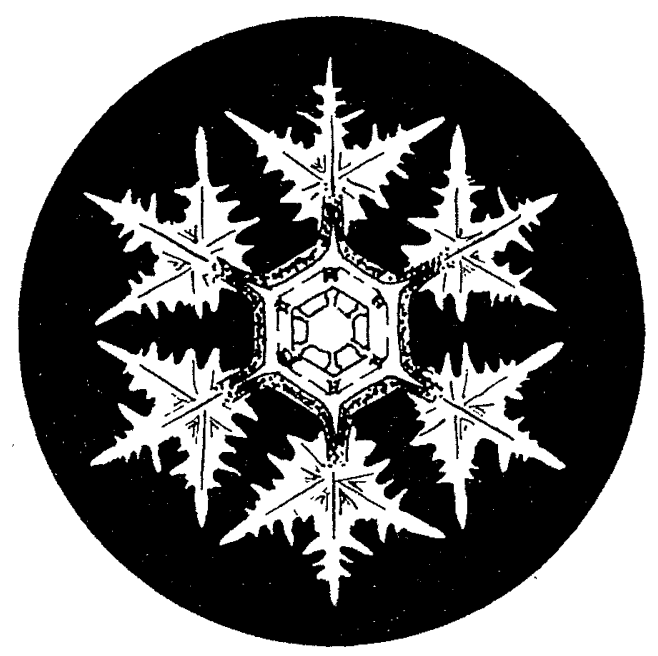

